\title{
Validação do tempo de mensuração da freqüência cardíaca após esforço submáximo a 50 e 80\%*
}

\author{
João Carlos Bouzas Marins ${ }^{1}$, Adriano Luiz², André Monteiro² e Geraldo de Jesus ${ }^{2}$
}

\section{RESUMO}

O objetivo deste estudo foi de verificar a existência de diferença significativa entre a frequiência cardíaca de recuperação medida em diferentes intervalos de tempo após o esforço (respectivamente $6,10,15,20,25,30,35,40,45,50,55$ e 60 segundos) e a frequiência cardíaca de esforço.

Foram avaliados 26 indivíduos voluntários na faixa etária entre 19 e 26 anos, sendo todos do sexo masculino, não atletas, com e sem prática de exercícios físicos e não fumantes. A coleta de dados foi realizada no Laboratório de Performance Humana do Departamento de Educação Física - UFV (Viçosa - MG). A instrumentação utilizada foi constituída de um cicloergômetro marca Moviment 2000, para a realização do esforço, além do cardiotacômetro Polar Acurex para mensuração da freqüência cardíaca.

O protocolo desenvolvido inclui a mensuração da curva de recuperação da freqüência cardíaca durante um minuto, após a realização de um exercício em nível submáximo, caracterizando um tempo de 10 minutos em steady state em duas situações: (a) $50 \%$ e (b) $80 \%$. Para o cálculo da freqüência cardíaca-alvo (FCT) levou-se em consideração a seguinte fórmula proposta por Karvonen: FCT = \% (FCMax FCRep) + FCRep. Após os 10 minutos de steady state o avaliador era orientado a interromper completamente o exercício, iniciando um processo passivo de recuperação, sendo

* Trabalho realizado no Laboratório de Performance Humana, Departamento de Educação Física, Universidade Federal de Viçosa.

1. Professor Assistente da UFV; Mestre em Biociências da Atividade Física UFRJ.

2. Pesquisador Júnior do LAPEH - Laboratório de Performance Humana UFV.

Endereço para correspondência:

Universidade de Murcia

Facultad de Biología

Departamento de Fisiología y Farmacología

Campus Espinardo

Bec. João Carlos Bouzas Marins

30.100 - Murcia

España

Correio eletrônico: jcbouzas@ fcu.um.es considerada a última FC registrada durante o esforço e em seguida registrado os valores da $\mathrm{FC}$ nos seguintes intervalos pós-esforço: $6,10,15,20,25,30,35,40,45,50,55$ e 60 segundos.

O tratamento estatístico incluiu a análise de variância (Estatística - Levin, J.) entre a média de cada mensuração da FC e o tempo zero (última FC registrada ao término da atividade submáxima no cicloergômetro).

Os resultados apurados apontaram como não significativa $(\mathrm{p}<0,05)$ a diferença dos valores obtidos apenas para a parcial de 6 segundos, tanto para o esforço a 50 e $80 \%$, sendo considerada significativa a diferença dos valores obtidos da FC a partir dos 10 segundos de mensuração.

Com os resultados obtidos neste estudo, é possível concluir que o registro ideal da FC pós-esforço submáximo de 503 $80 \%$ da FCM deverá compreender um tempo de aproximadamente 6 segundos. A parcial de 10 segundos, apesar de haver sido considerada, estatisticamente, como significativamente diferente, apresentou em valores absolutos uma redução que em termos práticos não interfere no planejamento da curva de intensidade. A coleta da FC, utilizando a parcial de 15 segundos, geralmente empregada por boa parte dos preparadores físicos, freqüentemente poderá induzir a erro de interpretação da intensidade proposta pelo exercício, o que, por sua vez, modificará a planificação do treinamento de forma incorreta.

Palavras-chave: Treinamento desportivo. Freqüência cardíaca. Prescrição de exercícios.

\section{ABSTRACT \\ Validation of the measuring time of the heart rate after sub- maximal physical exercise at $50 \%$ and $80 \%$}

The purpose of this study was to evaluate the existence of significant different between the recovery heart rate, measured at different intervals of time after physical exercise $(6,10,15$, $20,25,30,35,40,45,50,55$, and 60 seconds, respectively) and the exercise heart rate.

Twenty-six voluntary subjects at ages varying from 19 to 26 years were evaluated. All were male, non-athletes, nonsmokers, with or without regular practice of physical exercise. The data were collected at the Laboratory of Human 
Performance of the Department of Physical Education, at the University of Viçosa. State of Minas Gerais, Brazil. The instrument used for physical effort was "Movimento 2000" cycloergometer and "Polar Acurex" cardiotachometer was used for heart rate measurements. The protocol included the measurement of the heart rate recovery curve during one minute after a bout of submaximal exercise, characterizing a time of 10 minutes at steady state in two situations: (a) 50\% and (b) $80 \%$. For the target heart rate (THR) calculation, the following formula proposed by Karvonen was used: THR $=\%$ (Max$H R-$ RestHR) + RestHR. After 10 minutes of steady state, the subject was instructed to interrupt the exercise completely, starting a passive process of recovery. The last HR during effort and the HR at the following intervals after effort were recorded: $6,10,15,20,25,30,35,40,45,50,55$, and 60 seconds.

The only non-significant result ( $p>0.05$ ) obtained was for the 6-second partial measurement, both for the effort at 50\% and 80\%; the differences of the values obtained for the HR from the 10-second partial measurement and beyond it were significant.

With the results obtained in this study, it is possible to conclude that the ideal recording of the HR after a submaximal exercise at 50 and $80 \%$ of MaxHR should encompass a time of approximately 6 seconds. The 10-second partial measurement, albeit considered statistically significant and different, presented in absolute values a reduction that in practice does not interfere in the planning of the intensity curve. The collection of HR data using the 15-seconds partial measurement, generally used by most physical education instructors, will frequently induce misinterpretation as to the intensity proposed by the exercise, which will therefore modify incorrectly the planning of the training.

Key words: Sport training. Heart rate. Exercise prescription.

\section{INTRODUÇÃO}

A busca da qualidade total vem sendo um objetivo em diversas áreas para se aprimorar ao máximo um determinado produto ou serviço. No treinamento físico este elemento implica na observância de todos os fatores relacionados com a capacidade de desenvolver a performance, principalmente se se tratar de um atleta. Visando maximizar o rendimento esportivo, estuda-se cada vez mais a questão do suporte nutricional e farmacológico ao atleta, a curva de recuperação física e psicológica após esforço, desenvolvimento de estratégias médico-desportivas preventivas para o surgimento de lesões, novos materiais de vestimenta ou elementos inerentes ao desporto como a bicicleta com menos peso e maior coeficiente aerodinâmico no ciclismo, bem como o desenvolvimento de novos métodos de treinamento físico, além da forma de controle de sobrecarga que representa um ponto de re- alce neste conjunto de fatores que interferem no rendimento físico.

Para se controlar a intensidade de trabalho é possível utilizar-se diversas estratégias como a curva de concentração de lactato, o limiar ventilatório, o consumo de oxigênio $\left(\dot{\mathrm{V}}_{2}\right)$, o índice de percepção de esforço (IPE) ou escala de Borg e, por fim, a freqüência cardíaca ${ }^{1}$. As três primeiras formas de controle de intensidade são excelentes instrumentos, porém no Brasil, devido ao alto custo de seu emprego, não são formas usuais de controle. O IPE, apesar de apresentar uma razoável correlação com os parâmetros fisiológicos já mencionados, é dependente de uma correta interpretação da escala por parte do praticante, sendo um parâmetro questionável para ser utilizado como único elemento de controle de trei$\mathrm{no}^{2}$.

A FC passa a ser o principal fator de controle de carga de treino, por sua alta correlação com o $\dot{\mathrm{VO}}_{2}{ }^{3}$, e principalmente por sua praticidade de mensuração via sistema de monitorização direta com o cardiotacômetro, ou por palpação.

A monitorização rotineira da FC propicia as seguintes vantagens: (a) um perfeito acompanhamento da intensidade de treino; (b) elemento de controle para o acompanhamento dos efeitos do treino; (c) viabiliza a elaboração de um planejamento objetivo com relação à carga de trabalho durante um micro, meso e macrociclo e (d) fornece um referencial se o atleta apresenta uma condição de $\operatorname{strain}^{1,4}$.

Nos últimos anos, com o surgimento de aparelhos pessoais de monitorização cardíaca (cardiotacômetro), revolucionouse o emprego deste parâmetro fisiológico de controle da intensidade do exercício físico. Contudo, o custo deste equipamento infelizmente não o torna acessível à maioria dos praticantes de exercício físico; sendo assim, a leitura por palpação de um pulso periférico torna-se a forma mais empregada no dia a dia de treinamento.

A técnica de palpação para a mensuração da FC compreende a contagem do número de batimentos através da palpação utilizando-se a ponta dos dedos médio e indicador sobre a artéria carótida, ou artéria radial, ou ainda no ápice do coração. O primeiro ponto de mensuração (artéria carótida) apresenta como principal vantagem a facilidade de percepção do batimento cardíaco, porém pode desencadear um efeito de bradicardia devido à compressão de estruturas baroceptoras, reduzindo significativamente a FC pós-exercício, induzindo, conseqüentemente, a um erro na avaliação da carga de traba1 ho ${ }^{5}$.

A mensuração da FC palpando-se o ápice do coração (ictus cordis) traz como principal limitação a percepção dificultosa quando o exercício é realizado com baixas FC. Já a mensuração da artéria radial não apresenta nenhum tipo de problema como resposta baroceptora, sendo de fácil localização e palpação, independentemente do ritmo cardíaco, sendo então a mais indicada quando do uso da técnica por palpação. Uma quarta técnica de palpação é apresentada por 
Katch \& McArdle ${ }^{6}$, ao indicarem o contato com a artéria temporal.

Para a mensuração da FC por palpação é necessário que o atleta interrompa sua atividade física para então fazer a tomada do pulso. Durante este intervalo de tempo desencadeia-se imediatamente uma redução do estímulo simpático e aumento da ação parassimpática do coração, trazendo, como conseqüência, uma redução da $\mathrm{FC}^{7}$. Dependendo do tempo gasto para a leitura da FC, obtém-se um registro totalmente inadequado ao que na realidade o praticante apresenta, induzindose a um erro de subestimação da intensidade aplicada. Entretanto existe uma faixa de tempo pós-esforço em que o efeito de redução da FC não é significativamente forte ao ponto de reduzir a FC a um nível tão diferente em comparação com a obtida durante o exercício, sendo esta faixa de tempo o alvo de investigação deste estudo.

\section{OBJETIVO}

O objetivo deste estudo foi de verificar a existência de diferença significativa entre $\mathrm{FC}$ de recuperação medida em diferentes intervalos de tempo após esforço (respectivamente 6, $10,15,20,25,30,35,40,45,50,55$ e 60 segundos) com a FC registrada de esforço submáximo a $50 \%$ e $80 \%$ da freqüência cardíaca máxima (FCM), levando-se em consideração a FC de reserva.

\section{METODOLOGIA}

O presente trabalho caracterizou-se por um estudo quase experimental tipo transversal para análise comparativa da FC pós-esforço medida em cicloergômetro. A amostra constituise de 26 indivíduos voluntários na faixa etária entre 19 e 26 anos, todos do sexo masculino, não atletas, com e sem prática de exercícios físicos e não fumantes.

A coleta de dados foi realizada no Laboratório de Performance Humana (LAPEH), localizado no Departamento de Educação Física da Universidade Federal de Viçosa, Viçosa Minas Gerais.

Foi utilizado um cicloergômetro da marca Moviment 2000 para as atividades de esforço, além de um cardiotacômetro da marca Polar (Accurex) para mensuração e controle da FC, bem como cronômetros da marca Sports Timer para controle dos estágios, assim como para os protocolos de recuperação.

O protocolo de avaliação foi realizado em dois momentos: no primeiro, o avaliado foi submetido a uma carga de trabalho de steady state 50\% da FCM, sendo o segundo momento com uma carga a $80 \%$ da FCM, separadas por um período de pelo menos uma semana.

Para o cálculo do valor da intensidade de trabalho com base na FCM optou-se por empregar a fórmula predita por Karvonen que leva em consideração a frequiência cardíaca de reserva (FCR); sendo assim, utilizou-se a seguinte equação ${ }^{8}$ :

$$
\text { FCT }=x .(\text { FCM }- \text { FCRep })+\text { FCRep }
$$

Onde:

FCT $=$ freqüência cardíaca de treino; $x=\%$ do esforço desejado; FCM =freqüência cardíaca máxima prevista; FCRep =freqüência cardíaca de repouso.

Para o cálculo da frequiência cardíaca máxima para destreinados foi empregada a fórmula preconizada por Sheffield ${ }^{9}$ :

$$
\mathrm{FCM}=205-(0,42 \times \text { idade })
$$

Cada elemento da amostra foi orientada a comparecer ao local de realização dos testes (total de dois 50 e $80 \%$ da FCM), com antecedência mínima de 15 minutos. O avaliador teve como procedimento para estabilização da FC de repouso equilibrada, induzir um período de repouso ao avaliado de pelo menos cinco minutos através da realização de uma entrevista para coleta de informações gerais; ao final desse tempo processava-se a coleta mensuração da FC de repouso para o cálculo da FC de treino. Posteriormente, o avaliado era encaminhado ao ergômetro, porém sem realização de exercícios por mais um período de cinco minutos.

Após o período de monitorização da FC na condição de repouso de dez minutos, a metodologia consistiu na realização do exercício no cicloergômetro por um tempo de cinco minutos com uma carga submáxima na qual se procedia o ajuste da carga em watts e velocidade de rotação suficientes para elevar a FC ao ponto preestabelecido a 50 ou $80 \%$ da FCM, além de atuar também como uma forma de aquecimento. Atingindo o ponto desejado, os avaliados foram submetidos a um período de steady state durante dez minutos com a carga já ajustada nos primeiros cinco minutos de exercício. Com o término da etapa de steady state (dez minutos, os avaliados eram orientados a interromper por completo o esforço, iniciandose então a coleta da FC de recuperação nos intervalos referentes a $6,10,15,20,25,30,35,40,45,50,55$ e 60 segundos, sendo esta coleta realizada com os indivíduos ainda sentados no cicloergômetro. $\mathrm{O}$ mesmo procedimento foi empregado nas duas sessões de avaliação com 50 e $80 \%$ de esforço submáximo.

O tratamento estatístico empregado foi através do estudo da análise de variância (Estatística - Levin, J.) entre a média de cada mensuração da FC e o tempo zero (última FC registrada ao término da atividade submáxima no cicloergômetro).

\section{APRESENTAÇÃO DOS RESULTADOS}

Os resultados apurados durante a mensuração da FC de recuperação com o primeiro tratamento estatístico 50\% da FCM em steady state são apresentados na figura 1 e quadro 1. Já os dados obtidos na realização do segundo tratamento estatístico 
(80\% da FCM em steady state) são apresentados na figura 2 e quadro 2 .

Com base no tratamento estatístico desenvolvido, foi possível constatar que não existe diferença significativa na mensuração pós-esforço apenas parcial seis segundos ao ser comparada no momento zero. Entretanto, após dez segundos e as demais mensurações até 60 segundos, encontrou-se um p > 0,005 .

\section{DISCUSSÃO DOS RESULTADOS}

O efeito da bradicardia logo após a interrupção do exercício e durante todo o transcorrer do tempo de avaliação ocorreu em ambas as situações (50 e 80\%), conforme o previsto por Astrand \& Rodahl ${ }^{10}$, Guyton ${ }^{11}$ e Brooks \& Fahey ${ }^{12}$, tendo como principal agente causal a inversão da ação neural entre as estruturas simpáticas e parassimpáticas que auxiliam na regulação da FC.

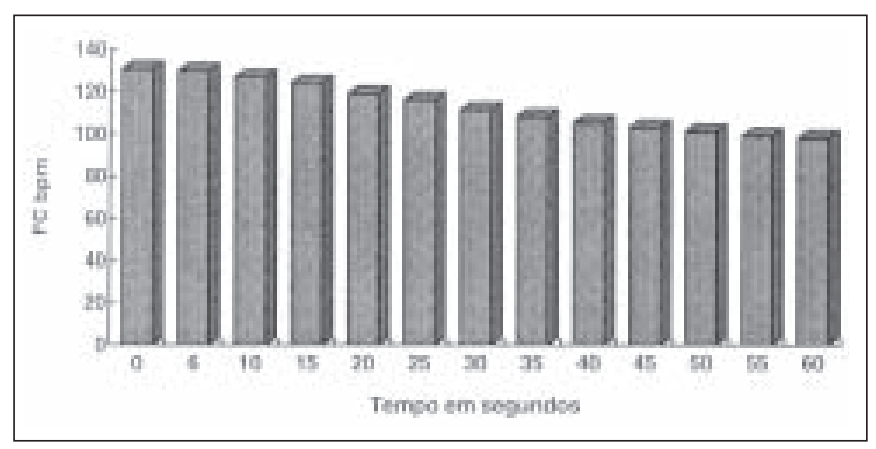

Fig. 1 - Comportamento da curva de recuperação da FC após um esforço submáximo a $50 \%$ da FCM

\section{QUADRO 1}

Comparação dos valores da FC entre o ponto zero (final do esforço a 50\%) e os tempos de mensuração pós-esforço (média \pm desvio-padrão)

\begin{tabular}{cccc}
\hline $\begin{array}{c}\text { Tempo de } \\
\text { mensuração } \\
\text { da FC (seg) }\end{array}$ & $\begin{array}{c}\text { Valores da FC } \\
\text { (bpm) nos tempos } \\
\text { de mensuração }\end{array}$ & $\begin{array}{c}\text { Valor da FC } \\
\text { (bpm) no tempo } \\
\mathbf{0 - 5 0 \%}\end{array}$ & Diferença \\
6 & $130 \pm 5,67$ & $131 \pm 4,63$ & NS \\
10 & $127 \pm 6,18$ & $131 \pm 4,63$ & $*$ \\
15 & $124 \pm 7,92$ & $131 \pm 4,63$ & $*$ \\
20 & $119 \pm 8,97$ & $131 \pm 4,63$ & $*$ \\
25 & $116 \pm 9,08$ & $131 \pm 4,63$ & $*$ \\
30 & $111 \pm 9,35$ & $131 \pm 4,63$ & $*$ \\
35 & $108 \pm 9,92$ & $131 \pm 4,63$ & $*$ \\
40 & $105 \pm 11$ & $131 \pm 4,63$ & $*$ \\
45 & $103 \pm 10,7$ & $131 \pm 4,63$ & $*$ \\
50 & $101 \pm 11$ & $131 \pm 4,63$ & $*$ \\
55 & $99 \pm 11,1$ & $131 \pm 4,63$ & $*$ \\
60 & $98 \pm 12,2$ & $131 \pm 4,63$ & $*$ \\
\hline
\end{tabular}

NS - Não significativo $(p<0,05)$ * Significativo.
A análise da curva de recuperação da FC na primeira situação-problema a 50\% permite observar que houve rápido retorno, em torno de 20 segundos, a uma FC base de recuperação a $120 \mathrm{bpm}$ propostos por Platonov ${ }^{13}$. Esta velocidade de recuperação foi influenciada pelo fato do estímulo empregado ser considerado de baixa intensidade. Na segunda situação-problema a $80 \%$ o patamar de 120 bpm não foi obtido no primeiro minuto de recuperação, havendo, conseqüentemente, necessidade de maior tempo de recuperação; este comportamento foi decorrente de um maior estímulo empregado, considerado como pesado por Wilmore \& Costill ${ }^{1}$.

No que tange ao principal ponto a ser investigado neste estudo, encontrou-se apenas na parcial de seis segundos, em ambas as situações de testagem, como não significativa a diferença observada. Contudo, para parcial de dez segundos a diferença registrada em termos absolutos correspondeu a -4 bpm para uma intensidade a $50 \%$ e $-3 \mathrm{bpm}$ a $80 \%$, o que representa de forma prática uma diferença a ser considerada

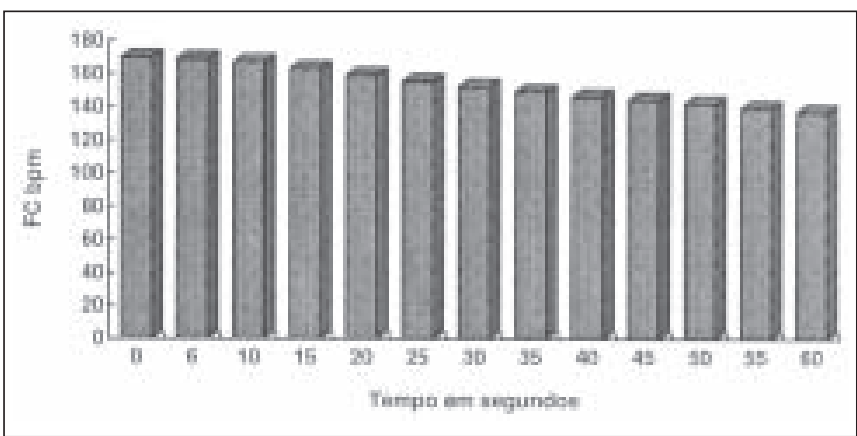

Fig. 2 - Comportamento da curva de recuperação da FC após um esforço submáximo a $80 \%$ da FCM

\section{QUADRO 2}

Comparação dos valores da FC entre o ponto zero (final do esforço a $80 \%$ ) e os tempos de mensuração pós-esforço (média \pm desvio-padrão)

\begin{tabular}{|c|c|c|c|}
\hline $\begin{array}{l}\text { Tempo de } \\
\text { mensuração } \\
\text { da FC (seg) }\end{array}$ & $\begin{array}{l}\text { Valores da FC } \\
\text { (bpm) nos tempos } \\
\text { de mensuração }\end{array}$ & $\begin{array}{c}\text { Valor da FC } \\
\text { (bpm) no tempo } \\
0-80 \%\end{array}$ & Diferença \\
\hline
\end{tabular}

6
10
15
20
25
30
35
40
45
50
55
60

$169 \pm 3,65$
$167 \pm 4,07$
$163 \pm 4,67$
$159 \pm 4,18$
$155 \pm 5,67$
$152 \pm 6,38$
$149 \pm 6,82$
$146 \pm 6,77$
$144 \pm 7,08$
$142 \pm 7,45$
$139 \pm 8,13$
$137 \pm 8,19$

NS

*

$170 \pm 3,23$

$170 \pm 3,23$

$170 \pm 3,23$

$170 \pm 3,23$

$170 \pm 3,23$

$170 \pm 3,23$

$170 \pm 3,23$

$170 \pm 3,23$

$170 \pm 3,23$

$170 \pm 3,23$

$170 \pm 3,23$ 


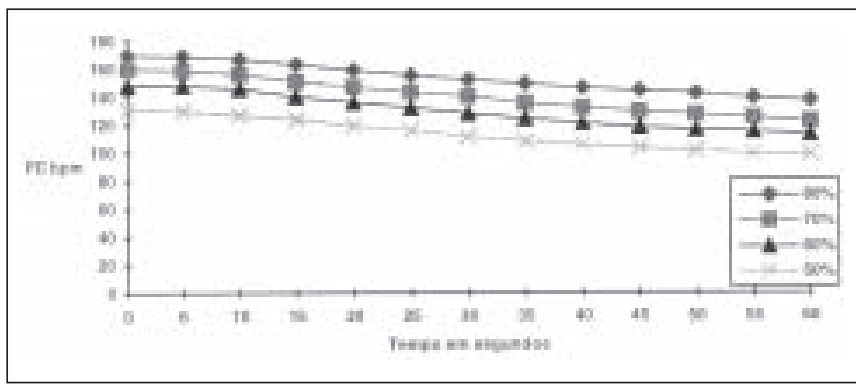

Fig. 3 - Comportamento da curva de recuperação da FC após um esforço submáximo a 50, 60, 70 e 80\% da FCM

por parte do preparador físico. Entretanto, para as parciais de 15 segundos a diferença observada foi de $-7 \mathrm{bpm}$ em ambas as situações, sendo este valor tanto estatisticamente como de forma prática sendo considerada como significativamente diferente.

Num estudo desenvolvido por Manso \& Marins ${ }^{14}$, aplicando uma metodologia semelhante para intensidades de $60 \mathrm{e}$ $70 \%$ da FCM, encontraram-se resultados próximos ao deste estudo. É importante destacar que a curva de recuperação mostrou ma tendência de deflexão independente da intensidade, porém cada uma assumindo uma faixa de FC diferenciada, independentemente do tempo analisado, como pode ser visto na figura 3 , que apresenta a curva de recuperação da FC após quatro intensidades diferenciadas de exercício.

$\mathrm{O}$ efeito da bradicardia com a conseqüente redução de batimentos também pode ser observado na figura 4 , ao comparar os resultados de Manso \& Marins ${ }^{14}$, com os resultados do presente estudo. Uma análise de ambos os estudos torna evidente que a redução da FC nas parciais seis e dez segundos não representam um valor que induza a um erro metodológico para o cálculo da intensidade de exercício. Contudo, nas parciais de 15 segundos em diante os valores tornam-se, tanto estatisticamente como de forma prática, significativamente diferentes em relação a FC de esforço, o que poderá induzir a um erro de avaliação da intensidade prescrita ao se subestimar o esforço realizado.

De acordo com as observações de Pollock et al. ${ }^{15}$, Cotton \& Dill ${ }^{16}$ e Manso \& Marins ${ }^{14}$, recomenda-se o registro da FC o mais rapidamente possível, dentro da faixa de seis a dez segundos, para se evitar a ocorrência de erros resultantes da desaceleração dos batimentos cardíacos.

A maior agilização da coleta da $\mathrm{FC}$, impõe a necessidade do preparador físico treinar o atleta para que este faça sua própria mensuração. Este procedimento deverá ser ensinado ao atleta desde o início de sua carreira esportiva, ou no início de cada temporada, utilizando-se um cardiotacômetro (preferencialmente) para se averiguar o nível de confiabilidade e agilização da mensuração por parte do praticante.

É fundamental uma alta precisão na tomada do dado, pois o erro de um batimento na técnica de seis segundos propiciará

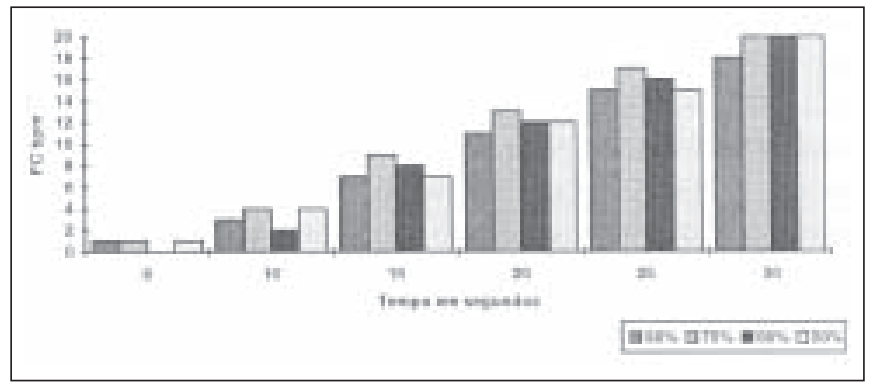

Fig. 4 - Valores médios de redução da FC em diversas parciais de tempo após quatro intensidades diferenciadas em steady state

na realidade um erro de $\pm 10 \mathrm{bpm}$, valor este maior que a tomada em 15 segundos. Já na mensuração em dez segundos o erro de contagem de um batimento implicará em um valor de $\pm 6 \mathrm{bpm}$. Assim, é fundamental para o praticante estar perfeitamente familiarizado com a aplicação desta técnica.

Caso o praticante apresente certa insegurança na contagem, a técnica de 15 segundos poderá induzir maior tranqüilidade de leitura, porém expõe a um erro de leitura que é considerado como significativo.

Cabe realçar que o tempo de leitura entre seis a dez segundos é indicado quando da interrupção do esforço para se realizar a leitura da FC. Em condições de repouso, ou durante um teste ergométrico em que existe a manutenção do estímulo elétrico do coração, pode-se perfeitamente mensurar com a técnica de 15 segundos na qual a margem de erro para a contagem é reduzida para $\pm 4 \mathrm{bpm}$, o que não altera de forma decisiva os resultados.

\section{CONCLUSÕES}

Os resultados apurados neste estudo permitem concluir que, estatisticamente, para uma condição de mensuração da freqüência cardíaca após uma atividade física em steady state a 50 e $80 \%$ da frequiência cardíaca máxima, levando em consideração a freqüência cardíaca de reserva, numa condição de repouso passivo, apenas os escores obtidos na parcial de seis segundos, foi considerada como não significativa a diferença. Porém os resultados absolutos obtidos na parcial de dez segundos em ambas as situações viabiliza sua adoção no dia a dia de trabalho, sem contudo induzir a um erro de planejamento da curva de intensidade de trabalho.

O uso da parcial de 15 segundos para mensuração da FC usualmente empregada por parte dos preparadores físicos, foi considerada como significativa sua diferença tanto estatisticamente quanto de forma absoluta, não sendo recomendada sua adoção, visto que poderá induzir uma falsa interpretação, por subestimar a intensidade de trabalho aplicada ao praticamente. Com base neste erro, o preparador físico irá propor um aumento na carga de trabalho, gerando assim uma sobrecarga e incrementando substancialmente o desgaste do 
atleta, visto que durante o exercício a FC estará acima da objetivada.

Deve-se ensinar aos atletas a realizarem suas próprias mensurações, a fim de abreviar o máximo possível o intervalo de tempo entre a interrupção do exercício e a leitura da FC.

A mensuração da FC pelo sistema individualizado com o uso do cardiotacômetro representa um avanço no controle da intensidade de treino, tornando seu registro mais fidedigno, sem sofrer interferência do reflexo de bradicardia que ocorre quando da interrupção do exercício para a leitura por apalpação.

\section{SUGESTÕES}

Com relação à realização de novas pesquisas sobre este tema, sugere-se executar o mesmo tipo de pesquisa em vários tipos de população: cardíacos, atletas de diversas modalidades, fumantes, idosos, entre outros. Existe também a possibilidade de análise da curva de recuperação em percentuais não utilizados nesta pesquisa como 90 e 100\%, além de uma forma de recuperação ativa, o que pode ou não alterar a curva de recuperação.

\section{REFERÊNCIAS}

1. Wilmore JH, Costill DL. Physiology of sports and exercise. Champaign: Human Kinetics, 1994.

2. Pollock ML, Wilmore J. Exercício na saúde e na doença. Rio de Janeiro: Medsi, 1993.
3. McArdle W, Katch F, Katch V. Fisiologia do exercício: energia, nutrição e desempenho humano. Rio de Janeiro: Guanabara Koogan, 1992.

4. Fox EL, Bowers RW, Foss ML. Bases fisiológicas da educação física e dos desportos. Rio de Janeiro: Guanabara Koogan, 1991.

5. White JR. EKG changes using the carotid artery for heart rate monitoring. Med Sci Sports 1977;9:88-94.

6. Katch F, McArdle W. Nutrição, exercício e saúde. Rio de Janeiro: Medsi, 1996.

7. A.C.S.M. Prova de esforço \& prescrição de exercício. Rio de Janeiro: Revinter, 1994.

8. Marins J, Giannichi R. Avaliação \& prescrição de atividade física: guia prático. Rio de Janeiro: Shape, 1996.

9. Sheffield L, Holt JH, Reevers TJ. Exercise graded by heart rate in electrocardiographic testing for angina pectoris. Circulation 1965;32:622 29.

10. Astrand P, Rodahl K. Tratado de fisiologia do exercício. Rio de Janeiro: Guanabara, 1986.

11. Guyton AC. Tratado de fisiologia médica. Rio de Janeiro: Guanabara, 1991.

12. Brooks J, Fahey T. Exercise physiology: human bioenergetics and its applications. New York: Mcmillan Publishing, 1984.

13. Platonov VN. El entrenamiento deportivo - Teoría y metodologia. Barcelona: Paidotribo, 1992

14. Manso EN, Marins JB. Estudo do comportamento da curva de recuperação da frequiência cardíaca após esforço submáximo. Rev Mineira Educ Física 1996;4:51-8.

15. Pollock M, et al. Validity of the palpation technique of heart rate determination and its estimation of training heart rate. Res Q 1972;43:77-81.

16. Cotton F, Dill D. On the relationship between the heart rate during exercise and that of immediate postexercise period. Am J Physiol 1983;54: 901-5. 